\title{
Immunocytochemical Localization of Peptidylarginine Deiminase Type III, Trichohyalin and Deiminated Trichohyalin in Infant Rat Dorsal Skin Hair Follicle
}

Takako Ohsawa, Akihito Ishigami, Kyoichi Akiyama and Hiroaki Asaga

Department of Bioactivity Regulation, Tokyo Metropolitan Institute of Gerontology, 35-2, Sakae-cho, Itabashi-ku, Tokyo 173-0015, Japan

(Received 26 March 2001, and accepted 16 May 2001)

\begin{abstract}
Peptidylarginine deiminase type III is a posttranslational enzyme to convert arginine residues in the proteins to citrullines, and exists specifically in the hair follicle and epidermis. We studied the immunocytochemical localization of the enzyme in the infant rat dorsal skin hair follicle, then compared it with the localization of trichohyalin, a putative target protein of the enzyme, and deiminated trichohyalin, the product of the enzyme reaction. The peptidylarginine deiminase type III was found to localize in both the Huxley's and Henle's layers of the inner root sheath, in the cuticle layer at the middle bulb region up to the middle follicle region, and also in the medulla cells closed to the dermal papilla and further up the follicle in the lower follicle region. The localization of trichohyalin was similar to that of the peptidylarginine deiminase type III, except that trichohyalin was detected as granules in the medulla cells closed to the dermal papilla and further up the follicle in the lower follicle region, and not in the cuticle layer. Two-dimensional gel electrophoresis revealed that the deiminated proteins in the hair follicle detected using a monospecific antibody to the chemically modified citrulline residues were deiminated trichohyalin and its proteolytic fragments. The deiminated trichohyalin detected as deiminated proteins by this antibody was found to localize in the inner root sheath from the upper bulb region further up the follicle, and also in the medulla at the middle follicle region and further up the follicle. These findings indicated that the trichohyalin and peptidylarginine deiminase type III were coincidentally expressed, but the deimination of trichohyalin occurred after their expression during the development of the hair follicle.
\end{abstract}

Peptidylarginine deiminases (PAD; EC 3. 5. 3. 15) are enzymes to convert arginine residues in protein to citrullines in the presence of $\mathrm{Ca}^{2+}$ ions, and recently classified into four isoforms of type I, type II, type III and type IV $(38,12,26)$. PAD

Correspondence to: Dr Takako Ohsawa at above address.

Tel: $+81-3-3964-3241(3067)$

Fax: +81-3-3579-4776

Email: ohsawa@tmig.or.jp is known to be widely distributed in vertebrate tissues: type I is restricted for the epidermis and uterus $(26,37)$, and also in the rat stomach $(12)$; type II is in the muscle, uterus, spinal cord, salivary glands, pancreas (37), and in the lung, spleen, kidney, stomach, brain and ovary (12), and also in the pituitary and thymus $(33,39)$; type III is in the hair follicle and epidermis (37), and also in the rat ovary (12); type IV is highly expressed in the pancreas, spleen, ovary, and less strongly in the liver, lung, stomach, kidney, 
uterus, dermis and weakly in the brain, heart and epidermis $(38,12)$. Although the function of PAD is still unclear, its physiological significance has been suggested by recent reports that the PAD in the brain is localized in the myelin sheath assembly sites (7), in the immature oligodendrocyte (2), and may have a key role in myelination and in multiple sclerosis (16). PAD in the epidermis is involved in the terminal differentiation of keratinocyte (29), and related to the psoriatic hyperproliferative epidermis (10). Furthermore, it has been reported that citrulline residues of filaggrin in the epidermis are essential in the epitopes to rheumatoid arthritis-specific autoantibodies $(27,6)$.

PAD in the hair follicle is classified as type III, and the cDNA has been cloned from sheep hair follicles (24), followed by cloning from the rat epidermis (17) or human skin (13). The immunocytochemical or in situ hybridization studies have revealed the localization of the PAD type III in the inner root sheath or medulla (24, 13). Trichohyalin $(\mathrm{THH})$, which is a filamentassociated protein of $\sim 200 \mathrm{kDa}$ found as a major component of the trichohyalin granules in the inner root sheath and medulla cells of the hair follicle $(25,19,34,23)$, has been reported to contain citrulline residues, indicating that THH is a target protein of the PAD type III (22). It has been reported that the recombinant THH changed its conformation with increasing efficacy to transglutaminase (TGase) 3 after the modification by PAD, and both the THH and TGase 3 were coincidentally localized in the inner root sheath and medulla $(35,36)$. These reports suggested that the THH granule is first deiminated by PAD which denatures it and makes it more soluble, which renders it available for efficient crosslinking by TGase 3 to form a highly cross-linked rigid structure in the inner root sheath or medulla cells.

Although the coincidental localization of $\mathrm{THH}$ and PAD type III $(24,13)$, or THH and TGase (36) have been reported, the localization of the deiminated $\mathrm{THH}$ has not been reported. In addition, it is not yet clear whether the deiminated protein in the hair follicle is only THH. Here we describe the immunocytochemical studies of the localization of the PAD type III and THH, as well as the deiminated $\mathrm{THH}$, and Western blot analysis of the deiminated proteins in the infant rat dorsal skin hair follicle.

\section{MATERIALS AND METHODS}

Antibodies. A monospecific antibody to the chemically modified citrulline (28) was provided by Dr T. Senshu (Tokyo Metropolitan Institute of Gerontology). A monoclonal antibody to THH (AE15) was kindly supplied by Dr T.-T. Sun (New York University School of Medicine, NY), and Dr M. Manabe (Akita University, Japan). For the preparation of a polyclonal antibody to the rat PAD type III, its partial sequence (residues 55-SMERNRERADTRR-67) (17) was synthesized using Fmoc (9fluorenylmethoxycarbonyl) chemistry with an additional cysteine residue at the terminal end for conjugation with the keyhole limpet hemocyanin. A rabbit was immunized with the conjugate and the antiserum was subjected to affinity purification using the immunized peptide. The purified antibody to PAD type III visualized a single band on the Western blot of the protein extract of the rat vibrissa follicles with $0.1 \mathrm{M}$ Tris- $\mathrm{HCl}$ ( $\mathrm{pH} 7.6$ ) containing $1 \mathrm{mM}$ phenylmethylsulfonyl fluoride (Sigma, MO, USA) and 0.1\% Nonidet P-40 (Daiichi Pure Chemicals, Japan) (Fig. 1). It did not show measurable reactions with any of the recombinant rat PAD type I and type IV (11), and the purified rat skeletal muscle PAD type II.

Western blotting. 7-day old Wistar rats were purchased from Japan SLC, Inc. (Shizuoka, Japan). After decapitation, the dorsal skins were spread on slide glasses with the epidermis sites up, and frozen immediately on dry ice. The frozen skins were scraped by a scalpel from the top of the epidermis to the dermis, and then the dermis, which was thought to be rich in hair follicles, was collected. The obtained frozen powder-like scrapes were extracted with Laemmli's lysis buffer (14), and subjected to two-dimensional gel electrophoresis using isoelectric focusing (IEF) (pH3.5-10) on 4\% polyacrylamide gel in the first dimension, and $7.5 \%$ polyacrylamide gel containing sodium dodecyl sulfate (SDS) in the second dimension (18). Western blotting to a nitrocellulose membrane and the detection of THH or the deiminated proteins were performed as previously described (28).

Immunocytochemistry. Small square pieces of the dorsal skin $(5 \times 5 \mathrm{~mm})$ were excised and fixed with $10 \%$ paraformaldehyde/PBS solution at $4^{\circ} \mathrm{C}$ for $4 \mathrm{~h}$. After sufficient washing with cold phosphate-buffered saline (PBS), the pieces were 


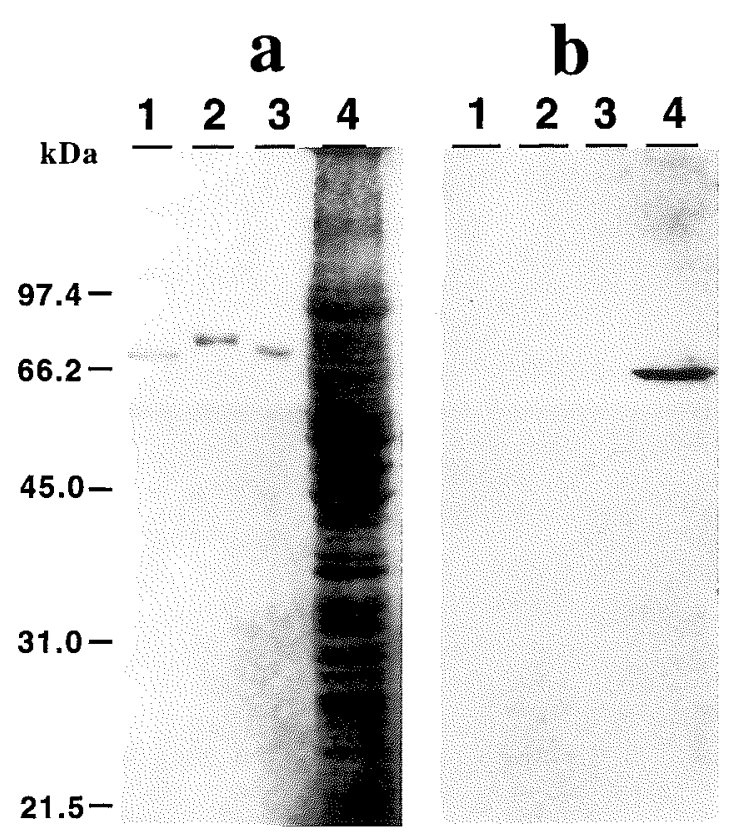

Fig. 1 Specificity of the antibody to rat PAD type III. Recombinant rat PAD type I (lane 1), PAD type II from rat skeletal muscle (lane 2 ), recombinant rat PAD type IV (lane 3), and the extract of rat vibrissa follicles (lane 4) were resolved by sodium dodecylsufate-polyacrylamide gel electrophoresis (SDS-PAGE) on 10\% polyacrylamide gel and Western blotted to the nitrocellulose membranes for amido black staining (a) and for immunoblotting with the antibody to rat PAD type III (b).

incubated with a series of sucrose solutions, embedded in O.C.T. compound 4583 (Miles Inc., USA), and cryosectioned to a $6 \mu \mathrm{m}$ thickness. For the immunostaining of the deiminated proteins, the specimen was pretreated to modify the citrulline residues as previously described (3). All stainings were performed using the appropriate combinations of antibodies and Vectastain ${ }^{\circledR} \mathrm{ABC}$ Kits (Vector Laboratories), and 3, 3'diaminobenzidine (DAB) as a chromogen. Counterstaining was performed using hematoxylin.

\section{RESULTS}

\section{Gel electrophoresis and immunoblotting}

To determine the deiminated proteins, the protein extract from the scrapes of the dermis enriched with hair follicles was subjected to twodimensional gel electrophoresis and Western blotting. The deiminated proteins were detected as two major and a few streaks, each supposed to be composed of closely packed charged isomers (Fig. 2-a). One of the major streaks (arrowhead 1) co-migrated with a similar streak visualized with AE15, and was identified as $210-\mathrm{kDa}$ THH (Fig. 2-b). The streak of the deiminated proteins showed a slightly curved anodic extension that was hardly detectable with AE15. The other major streak of the deiminated proteins showed a concave-shaped curve in the range from 121 to $205 \mathrm{kDa}$ with decreasing second dimensional mobility with increasing acidic properties. Its cathodic end appeared to meet the anodic end of a faint array of AE15-positive spots (arrowhead 2). In order to confirm whether the acidic components of the streaks of the deiminated proteins were derived from $\mathrm{THH}$, the protein extract from the dermis scrapes was incubated at $37^{\circ} \mathrm{C}$ for 30 min in the presence of $2 \mathrm{mM} \mathrm{Ca}^{2+}$ and $20 \mathrm{mM}$ cystamine, an inhibitor of endogenous TGase, and then applied to the gel electrophoresis and Western blotting. This caused extensive depletion of the AE15-positive signals leaving two major signals (Fig. 2-d). The less acidic signal was located on a thin line extending from a large smear near the upper anodic corner (arrow 1 in Figs. 2-c, -d). The AE15-positive signal was overlapped with a strong oblique signal of deiminated proteins distinguishable above the background smear (arrow 2 in Figs. 2-c, -d). These observations supported our idea of that the acidic deiminted proteins in the original extract were derived from THH.

\section{Immunocytochemistry}

Positive staining of the deiminated THH was observed in the Huxley's and Henle's layers of the inner root sheath, and the medulla in the middle follicle region and further up the follicle (Fig. 3-a). In the lower follicle region, the positive signals of the deiminated THH appeared to emerge from the Henle's layer and further up the follicle (Fig. 3-b). No positive staining of the medulla cells was observed in the lower follicle region near the bulb. THH was readily detectable in the inner root sheath in the lower bulb region, and in the medulla cells closed to the dermal papilla and further up the follicle in the lower follicle region (Fig. 3-d). The THH-positive signals in the inner root sheath persisted up to the cells at the middle bulb region, and then abruptly disappeared in the middle follicle region (Fig. 


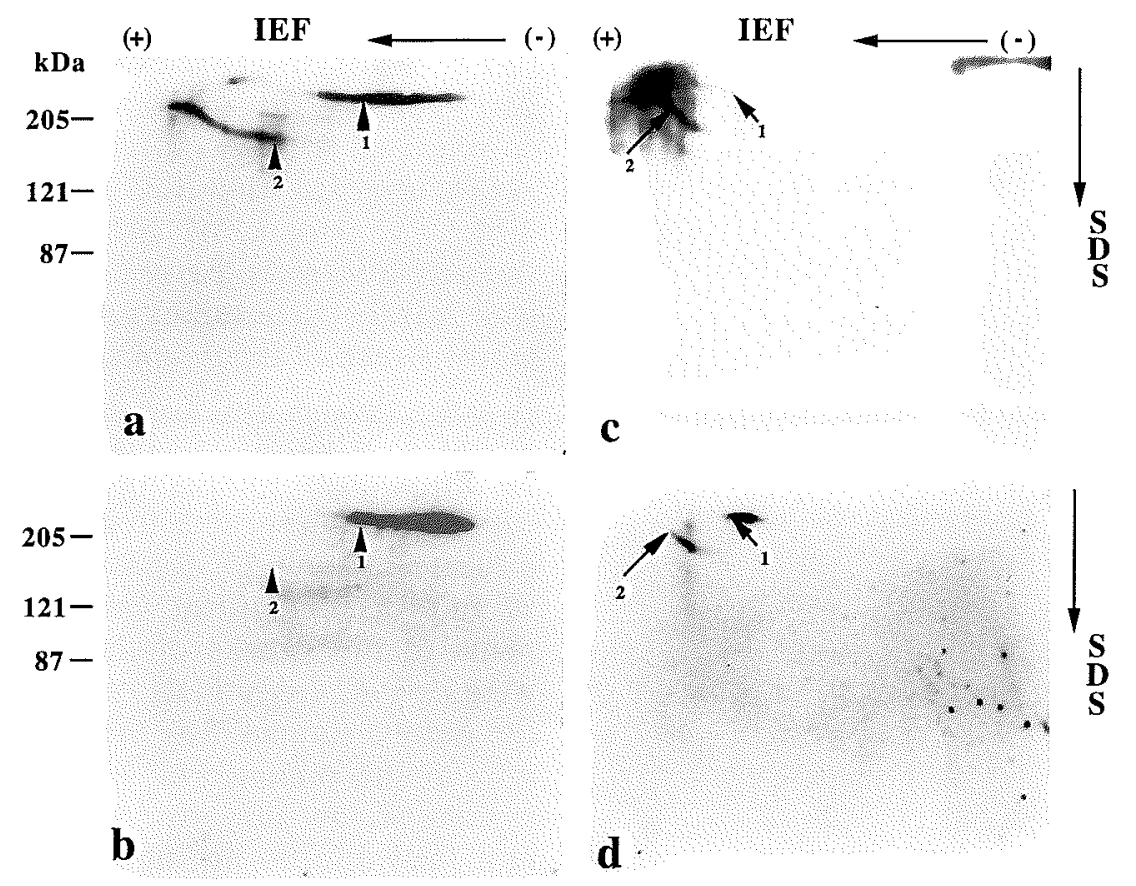

Fig. 2 Two-dimensional Western blotting of deiminated proteins and THH. The protein extracts from hair follicle-rich dermis scrapes were applied on two-dimensional gel electrophoresis and Western blotted for the detection of deiminated proteins (a, c), and THH $(a, c)$, before $(a, b)$ or after $(c, d)$ the incubation of the extract at $37^{\circ} \mathrm{C}$ for $30 \mathrm{~min}$ in the presence of $2 \mathrm{mM} \mathrm{Ca}^{2+}$ and $20 \mathrm{mM}$ cystamine as described in text. Numbered arrows and arrowheads show reference positions on each paired plate.

3-c). No THH-positive staining was observed in the medulla cells of the middle follicle region. The positive staining of PAD type III appeared to emerge in the Henle's and Huxley's layers of the inner root sheath and also in the cuticle layer of the middle bulb region and further up the follicle in the lower follicle region (Fig. 3-f). The positive staining of PAD type III was also detectable in the medulla cells closed to the dermal papilla and further up the follicle in the lower follicle region. In the middle follicle region, the positive signal of PAD type III in the inner root sheath disappeared, and no signal was detectable in the medulla cells (Fig. 3-e).

\section{DISCUSSION}

This study was aimed to clarify the localizations of PAD type III, THH and their reaction products of the deiminated THH in the hair follicle of the infant rat dorsal skin. The antibody used here to detect the deiminated proteins is a monospecific antibody to the citrulline residues. Hitherto, the deiminated proteins detected by this antibody were the deiminated forms of the keratins and filaggrin in the epidermis $(29,30,1)$, keratins in the keratinocyte (20), vimentin (4), myelin basic protein (2), and unidentified proteins in the rat brain damaged by perfusion with oxygen-deprived media (5). Therefore, we decided to identify the deiminated proteins in the hair follicle by Western blot analysis using twodimensional gel electrophoresis. The deiminated signals were detected as two major and a few minor curved streaks above the molecular range of $121 \mathrm{kDa}$, indicating that they were unlikely to be derived from the keratins. One of the major streaks was identified as $210-\mathrm{kDa}$ THH from the monoclonal antibody AE15, although its curved anodic extension could be hardly detected with AE15. The curved streak could be explained by a report that the deiminated recombinant $\mathrm{THH}$ peptide progressively decreased the mobility according to the increase in the deimination level (36). The other curved major streak showing a faster migration in the basic region appeared to have a cathodic extension overlapping a faint array of AE15-positive signals, and was suggested 


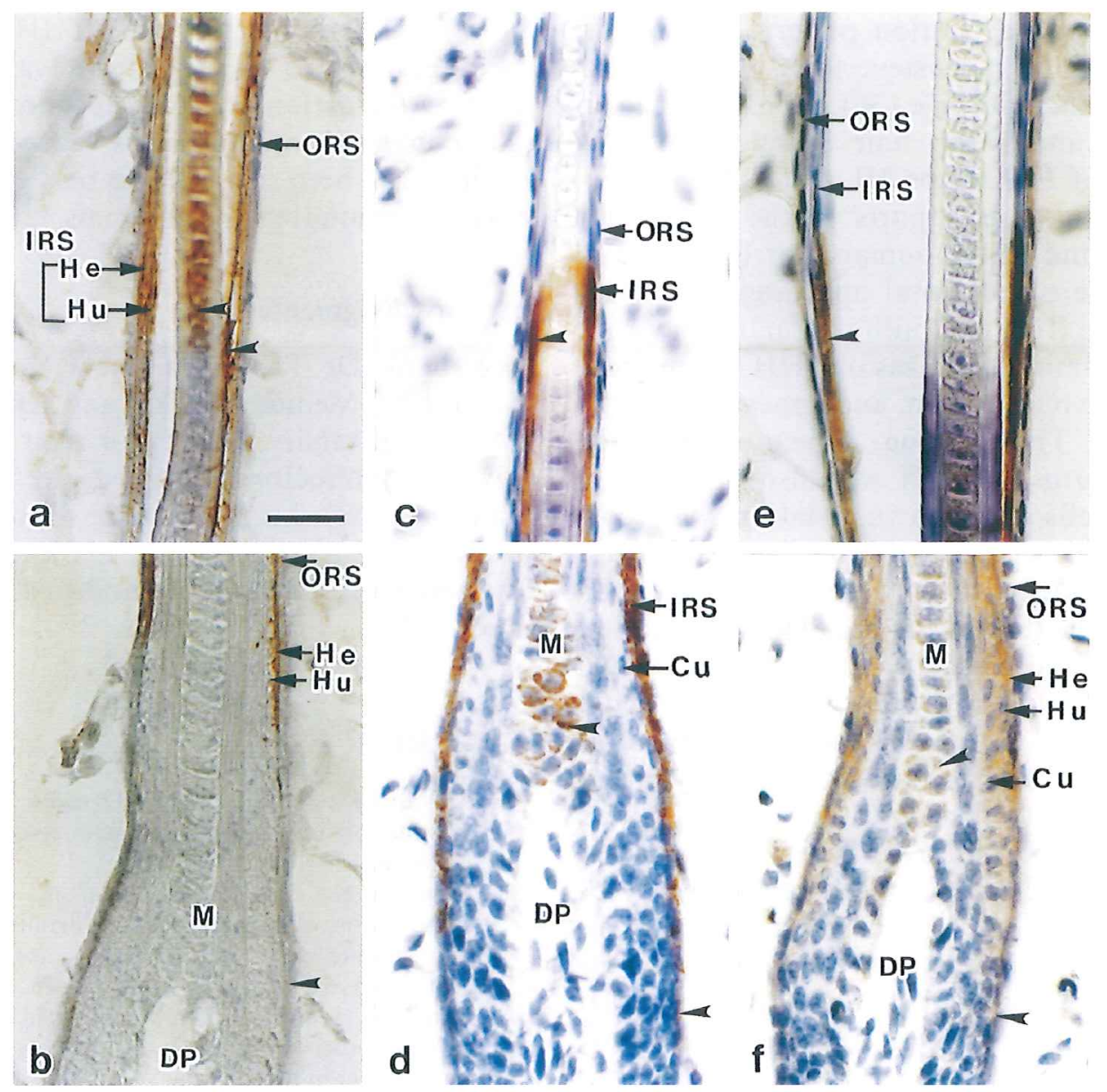

Fig. 3 Immunocytochemical localizations of deiminated proteins, trichohyalin (THH) and PAD type III in the hair follicle of infant rat back skin. The deiminated proteins were detected by chemical modification of citrulline residues following by probing with the monospecific antibody $(a, b)$, THH with the monoclonal antibody AE15 (c, d), and PAD type III with the affinity-purified polyclonal antibody (e, f) as described in the text. All sections were counterstained with hematoxylin. Middle follicle region (a, c, e), dermal papilla and lower follicle region (b, d, f). A relatively upper region of the hair follicle is shown in panel (a) to show intensely stained Henle's layer. Arrowheads show the positive signal. IRS, inner root sheath; He, Henle's layer; Hu, Huxley's layer; ORS, outer root sheath; M, medulla; DP, dermal papilla; Cu, cuticle layer. Scale bar, 25 $\mu \mathrm{m}$

to be the proteolytic products of $\mathrm{THH}$, whose presence has been reported $(25,19)$. However, the signal of AE15 was too weak to identify it as the proteolytic fragment of THH. If it is true that the deiminated $\mathrm{THH}$ or deiminated proteolytic fragments of THH could be the target of TGase, a TGase inhibitor might be effective for accumulation and make it easier to identify the deiminated $\mathrm{THH}$ or its fragments. We then incubated the extract of the hair follicle-rich dermis scrapes in the presence of $\mathrm{Ca}^{2+}$ and cystamine of an in- hibitor of TGase (31). The result showed that the two short streaks of the deiminated proteins in the very anodic region were overlapped with the two signals of THH in the Western blotting. These results indicated that the deimination of THH or its fragments was proceeded by the endogenous PAD and the deiminated forms were accumulated by the inhibition of the TGasemediated cross-linking. Thus it was concluded that the deiminated proteins in the hair follicle were derived from $\mathrm{THH}$ and its proteolytic frag- 
ments.

The observed localization pattern of THH in the hair follicle is consistent with other reports for the mouse hair follicle (36), human hair follicle $(19,13)$, and sheep hair follicle (24). Colocalization of PAD type III and THH is also consistent with other reports in the sheep hair follicle (24), and in the human hair follicle (13). In spite of the coincidental appearance of $\mathrm{THH}$ and PAD type III in the bulb region of inner root sheath, the deiminated forms of THH were hardly detected in the bulb region, and appeared over the bulb region. The lagging appearance of the deiminated forms of THH was also observed in the medulla cells in which vacuolation was recognized to appear, whereas both the THH and PAD type III coincidentally appeared in the medulla cells close to the dermal papilla. This observation might confirm the postulation that the deiminated THH might be cross-linked to large aggregates leaving a highly vacuolated structure (36). It is still unclear why the deimination does not occur coincidentally with the appearance of THH and PAD. However, it could be possible to assume that the cytosolic $\mathrm{Ca}^{2+}$ concentration might be increased slowly up to a level to trigger the reaction of $\mathrm{PAD}$. It was noticeable that both the positive staining of THH and PAD type III disappeared in the middle follicle region either in the inner root sheath or medulla, whereas the intense signal of the deiminated forms of $\mathrm{THH}$ appeared and continued further up the follicle. The reduction in the THH-positive staining in the middle follicle region has been reported by other investigators (36), and this could be explained by the fact that the deiminated $\mathrm{THH}$ and its fragments could be hardly detected on the Western blotting with AE15 as shown above. In addition, it could be considered that the deiminated forms of THH were cross-linked to the keratin-filaments at the middle follicle region, because it has been reported that AE15 reacts with THH in the granules but not with filament-associated THH (19).

In conclusion, it was found that the deiminated proteins in a hair follicle were $\mathrm{THH}$ and its proteolytic fragments, and they appear after the expression of THH and PAD type III. Probably the deiminated THH or its fragments bundle with keratin intermediate filaments (KIF) by the action of TGase 3 during the development of hair follicle in the upward direction, possibly in hardening the inner root sheath, and directing and anchoring the developing hair shaft, as already reported $(8,35,36,32)$. In medulla cells which contain few, if any, KIF (13), THH granules may be agglomerated during the upward differentiation by the action of PAD, and probably eventually converted to amorphous vacuolated deposits, which have been suggested to play critical roles in thermal regulation in mammals (21).

\section{Acknowledgments}

We thank Dr T.-T. Sun (New York University School of Medicine, NY) and Dr M. Manabe (Akita University, Japan) for their generous supply of a monoclonal antibody to THH (AE15), and Dr T. Senshu (Tokyo Metropolitan Institute of Gerontology, Japan) for his kind supply of a monospecific antibody to modified citrulline residues.

\section{REFERENCES}

1. Akiyama K., Senshu T.(1999) Dynamic aspects of protein deimination in developing mouse epidermis. Exp. Dermatol. 8, 177-186.

2. Akiyama K., Sakurai Y., Asou H., Senshu T.(1999) Localization of peptidylarginine deiminase type II in a stage-specific immature oligodendrocyte from rat cerebral hemisphere. Neurosci. Lett. 274, 53-55.

3. Asaga H., Senshu T.(1993) Combined biochemical and immunocytochemical analysis of postmortem protein deimination in the rat spinal cord. Cell Biol. Intt. 17, 525532.

4. Asaga H., Yamada M., Senshu T.(1998) Selective deimination of vimentin in calcium ionophore-induced apoptosis of mouse peritoneal macrophages. Biochem. Biophys. Res. Commun. 243, 641-646.

5. Asaga H., Ishigami A.(2000) Protein deimination in the rat brain: generation of citrulline- containing proteins in cerebrum perfused with oxygen-deprived media. Biomed Res. 21, 197-206

6. Girbal-Neuhauser E., Durieux J-J., Arnaud M., Dalbon P., Sebbag M., Vincent C., Simon M., Senshu T., MassonBessière C., Jolivet-Reynaud C., Jolivet M., Serre G.(1999) The epitopes targeted by the rheumatoid arthritisassociated antifilaggrin autoantibodies are postranslationally generated on various sites of (pro) filaggrin by deimination of arginine residues. $J$. Immunol. 162, 585594.

7. Gold MG., Freund CM., Palmer F., Feinstein DL.(2000) Messenger RNAs located in myelin sheath assembly sites. J. Neurochem. 75, 1834-1844.

8. Harding H. W. J., Rogers G. E.(1971) $\varepsilon$-( $\gamma$-glutamyl) lysine cross-linkage in citrulline- containing protein fractions from hair. Biochemistry 10, 624-630.

9. Heid H. W., Moll I., Franke W. W.(1988) Patterns of expression of trichocytic and epithelial cytokeratins in mammalian tissues. I. Human and bovine hair follicles. Differentiation 37, 137-157.

10. Ishida-Yamamoto A., Senshu T., Takahashi H., Akiyama 
K., Nomura K., lizuka H.(2000) Decreased deiminated keratin $\mathrm{Kl}$ in psoriatic hyperproliferative epidermis. $J$. Invest. Dermatol. 114, 701-705.

11. Ishigami A., Kuramoto M., Yamada M., Watanabe K., Senshu T.(1998) Molecular cloning of two novel types of peptidylarginine deiminase cDNAs from retinoic acidtreated culture of a newborn rat keratinocyte cell line. FEBS Lett. 433, 113-118.

12. Ishigami A., Asaga H., Ohsawa T., Akiyama K. Maruyama N.(2001) Peptidylarginine deiminase type I, type II, type III and type IV are expressed in rat epidermis. Biomedical Res.(22, 63-65).

13. Kanno T., Kawada A., Yamanouchi J., Yosida-Noro C., Yoshiki A., Shiraiwa M., Kusakabe M., Manabe M., Tezuka T., Takahara H.(2000) Human peptidylarginine deiminase type III: molecular cloning and nucleotide sequence of the cDNA, properties of the recombinant enzyme, and immunohistochemical localization in human skin. J. Invest. Dermatol. 115, 813-823.

14. Laemmli U. K.(1970) Cleavage of structural proteins during the assembly of the head of bacteriophage T4. Nature 227, 680-5.

15. Lee S-C., Kim I-G., Marekov L. N., O'Keefe E. J., Parry D. A. D., Steinert P. M.(1993) The structure of human trichohyalin. Potential multiple roles as a functional EF-handlike calcium-binding protein, a cornified cell envelope precursor, and an intermediate filament-associated (crosslinking) protein. J. Biol. Chem. 268, 12164-12176.

16. Mastronardi F. G., Mak B., Ackerly C. A., Roots B. I., Moscarello M. A.(1996) Modifications of myelin basic protein from multiple sclerosis. J. Clin. Invest. 97, 349358.

17. Nishijo T., Kawada A., Kanno T., Shiraiwa M., Takahara H.(1997) Isolation and molecular cloning of epidermaland hair follicle-specific peptidylarginine deiminase (Type III) from rat. J. Biochem. 121, 868-875.

18. O'Farrell PH.(1975) High resolution two-dimensional electrophoresis of proteins. J. Biol. Chem. 250, 4007-4021.

19. O'Guin W. M., Sun T.-T., Manabe M.(1992) Interaction of trichohyalin with intermediate filaments: three immunologically defined stages of trichohyalin maturation. J. Invest. Dermatol. 98, 24-32.

20. Ohsawa T., Maruyama I., Senshu T.(1999) Collateral occurrence of deimination of keratins with differentiation of an immortalized newborn rat keratinocte cell line cultured at air-liquid interface. J. Dermatol. Sci. 19, 68-73.

21. Rogers G. E.(1964) Structural and biochemical features of the hair follicle. In: Epidermis (Montagna W., Lobtig W. eds) Academic Press Inc., New York, 179-236.

22. Rogers G. E., Harding H. W., Llewellyn-S. I. J.(1977) The origin of citrulline-containing proteins in the hair follicle and the chemical nature of trichohyalin, an intracellular precursor. Biochim. Biophys. Acta 495, 159-75.

23. Rogers G. E., Fietz M. J., Fratini A.(1991) Trichohyalin and matrix proteins. Ann. N. Y. Acad. Sci. 642, 64-81

24. Rogers G., Winter B., McLaughlan C., Powell B., Nesci T. (1996) Peptidylarginine deiminase of the hair follicle: characterization, localization, and function in keratinizing tissues. J. Invest. Dermatol. 108, 700-707.

25. Rothnagel J. A., Rogers G. E.(1986) Trichohyalin, an intermediate filament-associated protein of hair follicle. $J$.
Cell Biol. 102, 1419-1429.

26. Rus'd A. A., Ikejiri Y., Ono H., Yonekawa T., Shiraiwa M., Kawada A., Takahara H.(1999) Molecular cloning of cDNAs of mouse peptidylarginine deiminase type I, type III and type IV, and the expression pattern of type $I$ in mouse. Eur, J. Biochen. 259, 660-669.

27. Schellekens G. A., de Jong B. A. W., van den Hoogen F. H. J., van de Putte L. B. A.(1998) Citrulline is an essential constituent of antigenic determinants recognized by rheumatoid arthritis-specific autoantibodies. J. Clin. Invest. 101, 273-81.

28. Senshu T., Sato T., Inoue T., Akiyama K., Asaga H.(1992) Detection of citrulline residues in deiminated proteins on polyvinylidene difluoride membrane. Anal. Biochem. 203, 94- 100.

29. Senshu T., Akiyama K., Kan S., Asaga H., Ishigami A., Manabe M.(1995) Detection of deiminated proteins in rat skin: probing with a monospecific antibody after modification of citrulline residues. J. Invest. Dermatol. 105, 163169.

30. Senshu T., Kan S., Ogawa H., Manabe M., Asaga H.(1996) Preferential deimination of keratin $\mathrm{Kl}$ and filaggrin during the terminal differentiation of human epidermis. Biochem. Biophys. Res. Commun. 225, 712-9.

31. Siefring G. E., Jr, Apostol A. B., Velasco P. T., lorand L. (1978) Enzymatic basis for the $\mathrm{Ca}^{2}+$-induced crosslinking of membrane proteins in intact human erythrocytes. Biochemistry 17, 2598-2604.

32. Strale W. E.(1965) Root sheath-dermal papilla relationships and the control of hair growth. In: Biology of the Skin and Hair Growth (Lyne A. G., Short B. F. eds). Angus and Robertson Ltd., Sydney, 35-57.

33. Sugawara K., Oikawa Y., Ouchi T. (1982) Identification and properties of peptidylarginine deiminase from rabbit skeletal muscle. J. Biochem.(Tokyo) 91, 1065-1071.

34. Sugiyama S.(1989) Cytochemistry of trichohyalin granules: a possible role for cornification of inner root sheath in the hair follicle. J. Dermatol. 16, 89-97.

35. Taresa E., Marekov L. N., Mei G., Melino G., Lee S.-C., Steinert P. M.(1996) Protein unfolding by peptidylarginine deiminase. Substrate specificity and structural relationships of the natural substrates trichohyalin and filaggrin. J. Biol. Chem. 271, 30709-30716.

36. Taresa E., Marekov L. N., Andreoli J., Idler W. W., Candi E., Chung S., Steinert P. M.(1997) The fate of trichohyalin. Sequential post-translational modifications by peptidylarginine deiminase and transglutaminases. J. Biol. Chem. 272, 27893-27901.

37. Terakawa H., Takahara H., Sugawara K.(1991) Three types of mouse peptidylarginine deiminase: characterization and tissue distribution. J. Biochem.(Tokyo) 110, 661666.

38. Yamakoshi A., Ono H., Nishijyo T., Shiraiwa M., Takahara H.(1998) Cloning of cDNA encoding a novel isoform (type IV) of peptidylarginine deiminase from rat epidermis. 1386, 227-231.

39. Watanabe K., Akiyama K., Hikicti K., Ohtsuka R., Okuyama A., Senshu T.(1998) Combined biochemical and immunochemical comparison of peptidylarginine deiminases present in various tissues. Biochim. Biophys. Acta 966, 375-83. 